Our Nature (2010) 8: 336-354

\title{
Cyanobacteria of Nepal: A Checklist with Distribution
}

\author{
S.K. Rai ${ }^{{ }^{*}}$, R.K. Rai ${ }^{2}$ and S. Jha ${ }^{1}$ \\ ${ }^{1}$ Department of Botany, P.G. Campus, T.U., Biratnagar, Nepal \\ ${ }^{2}$ Department of Botany, M.M.A.M. Campus, T.U., Biratnagar, Nepal \\ *E-mail: shivarai2003@yahoo.com
}

Received: 19.03.2010, Accepted: 23.07.2010

\begin{abstract}
This compilation work presents an up-to-date list of the cyanophycean algae reported by various workers in past from Nepal. It comprises a total 274 taxa belonging to 61 genera under 12 families.
\end{abstract}

Key words: Cyanobacteria, blue-green algae, enumeration, checklist, Nepal

\section{Introduction}

Nepal $\left(26^{\circ} 22^{\prime}-30^{\circ} 27^{\prime} \mathrm{N}, 80^{\circ} 04^{\prime}-88^{\circ} 12^{\prime} \mathrm{E}\right)$, a small Himalayan country (area ca 147181 $\mathrm{km}^{2}$ ), is very rich in water resources as it has about 2323 glacier lakes and 2252 snow fed rivers in the Himalaya; about 6000 rivers, streams and thousand of waterfalls in the mountain; and many lakes, ponds, puddles and wetlands in the terai regions of the country; altogether about $7437 \mathrm{~km}^{2}(5 \%$ of the total land) area as the favourite habitats for algae. Besides these, the diverse topography (elevation range $56 \mathrm{~m}$ to 8848 m) and climate also make the country rich in algal diversity. However, except sporadic collections, the extensive exploration of algae in the country has never been done as it has been given least preference in comparison to land vegetation specially the phanerogams.

This is an attempt to list out the total blue-green algae reported from Nepal by various phycologists at different time intervals till the date mainly on the basis of available literature. It includes a total 274 taxa as the latest data on the cyanophycean algae of Nepal. Here, the families are arranged following Desikachary (1959) and taxa are arranged alphabetically in each family.

\section{Checklist with distribution}

Family: Chroococcaceae Nägeli (1849)

1. Aphanocapsa anodontae Hansg.: Mingled with mosses on a brick wall at Balaju water garden, $1300 \mathrm{~m}$, Kathmandu (Watanabe and Komarek, 1988).

2. Aphanocapsa biformis A.Br.: Madhuban and Kusaha, Sunsari (Jha and Kargupta, 2001).

3. Aphanocapsa delicatissima W. West et G.S. West: A stream at Pisang, $3100 \mathrm{~m}$, Manang (Hirano, 1955).

4. Aphanocapsa elachista W. West et G.S. West var. conferta W. West et G.S. West: Khageri Khola at Tikauli, Chitwan (Das and Verma, 1996).

5. Aphanocapsa elachista W. West et G.S. West var. planctonica Smith: A glacier lake at Langtang Himal, 3700 m, Rasuwa (Hirano, 1969). 
6. Aphanocapsa grevillei (Hass.) Rabenh.: A pond at Luitel Bhanjyang, 770 m, Gorkha (Hirano, 1955); Langtang river, $2000 \mathrm{~m}$, Rasuwa (Hirano, 1969); mingled with mosses on a brick wall at Balaju water garden, $1300 \mathrm{~m}, \quad$ Kathmandu (Watanabe and Komarek, 1988); a pond at Mahendra Morang Campus, $72 \mathrm{~m}$, Biratnagar, Morang (Rai and Misra, 2010).

7. Aphanocapsa cf. muscicola (Menegh.) Wille: Sagarmatha National Park, Solukhumbu (Komarek and Watanabe, 1998).

8. Aphanocapsa pulchra (Kütz.) Rabenh.: Planktonic in stagnant water of Pimbahal pond at Pimbahal, 1300 m, Lalitpur (Joshi, 1979); a pond at Godawari, Lalitpur (Shrestha and Manandhar, 1983).

9. Aphanocapsa rivularis (Carm.) Rabenh.: Khair Khola at Tandi, Chitwan (Das and Verma, 1996).

10. Aphanocapsa roeseana De Bary: On damp bank by roadside at Godawari, 1400 m, Lalitpur (Watanabe and Komarek, 1988); planktonic in a pond near Sarda dam, Mahendranagar, Kanchanpur (Habib, 1997).

11. Aphanothece castagnei (Bréb.) Rabenh.: A glacial lake at Rarkya pass, $4850 \mathrm{~m}$, Manang (Hirano, 1963); Under a cliff with dripping water at Phakdingma, $2700 \mathrm{~m}$, Sagarmatha National Park, Solukhumbu (Watanabe and Komarek, 1994).

12. Aphanothece microscopica Näg.: A glacial lake at Langtang Himal, 3700 m, Rasuwa (Hirano, 1969); roadside ditches at Mahendranagar, Kanchanpur (Habib, 1997).
13. Aphanothece naegelii Wartm.: On damp bank by roadside at Godawari, 1400 m, Lalitpur (Watanabe and Komarek, 1988); rice field at Phoksiltar, 400 m, near Sun Koshi river, Udayapur (Rai and Misra, 2010).

14. Aphanothece cf. nidulans Richt. in Wittr. et Nordst.: Under a cliff with dripping water at Phakdingma, 2700 $\mathrm{m}$, Sagarmatha National Park, Solukhumbu (Watanabe and Komarek, 1994).

15. Aphanothece pallida (Kütz.) Rabenh.: Langtang river, $2000 \mathrm{~m}$, Rasuwa (Hirano, 1969); mingled with mosses on a brick wall at Balaju Water Garden, 1300 m, Kathmandu (Watanabe and Komarek, 1988).

16. Aphanothece stagnina (Spreng.) A.Br.: Floating on Rara lake, 2970 m, Mugu (Watanabe, 1995).

17. Chroococcus hansgirgi Schmidle: Madhuban and Kusaha, Sunsari (Jha and Kargupta, 2001).

18. Chroococcus limneticus Lemm.: Lower valley below Namchebazar along different habitats and smaller streams flowing into Dudh Koshi river, $2900 \mathrm{~m}$ to $4200 \mathrm{~m}$, Solukhumbu (Subba Raju and Suxena, 1979); on moist soil at Kirtipur, $1300 \mathrm{~m}$, Kathmandu (Shrestha and Manandhar, 1983).

19. Chroococcus limneticus Lemm. var. distans G.M. Smith: Chandi river at Chandranigahapur, Rautahat (Sahay et al., 1993).

20. Chroococcus minor (Kütz.) Näg.: On damp bank by roadside at Godawari, 1400 m, Lalitpur (Watanabe and Komarek, 1988). 
21. Chroococcus minutus (Kütz.) Näg.: A stream at Tukucha moor, $2600 \mathrm{~m}$, Mustang (Hirano, 1955); lower valley below Namchebazar along different habitats and smaller streams flowing into Dudh Koshi river, $2900 \mathrm{~m}$ to 4200 m, Solukhumbu (Subba Raju and Suxena, 1979); Chandi river and Bakaya river at Chandranigahapur, Rautahat (Sahay et al., 1993); a shallow lake near Kongma La, 5300 m, Sagarmatha National Park, Solukhumbu (Watanabe and Komarek, 1994); on a dead tree in the south shore of Rara lake, $2970 \mathrm{~m}$, Mugu (Watanabe, 1995); on decaying substratum in a ditch at Narayanghat, Chitwan (Das and Verma, 1996); Madhuban and Kusaha, Sunsari (Jha and Kargupta, 2001); Titrigachi pond at Koshi Tappu, 206 m, Kusaha, Sunsari (Rai and Misra, 2010).

22. Chroococcus schizodermaticus West: A pond at Mechi Campus, $93 \mathrm{~m}$, Bhadrapur, Jhapa (Rai and Misra, 2010).

23. Chroococcus tenax (Kirchn.) Hieron.: Chandi river at Chandranigahapur, Rautahat (Sahay et al., 1993); under a cliff with dripping water at Phakdingma, 2700 $\mathrm{m}$, Sagarmatha National Park, Solukhumbu (Watanabe and Komarek, 1994).

24. Chroococcus turgidus (Kütz.) Näg.: A stream at Tukucha moor, $2600 \mathrm{~m}$, Mustang; A pond at Pisang, $3100 \mathrm{~m}$ and Thaple Himal, 4000 m, Manang (Hirano, 1955); Chandi river at Chandranigahapur, Rautahat (Sahay et al., 1993).

25. Chroococcus cf. turicensis (Näg.) Hansg.: A shallow lake near Kongma
La, $5300 \mathrm{~m}$, Sagarmatha National Park, Solukhumbu (Watanabe and Komarek, 1994).

26. Chroococcus varius A.Br. in Rabenh.: Under a cliff with dripping water at Phakdingma, $2700 \mathrm{~m}$, Sagarmatha National Park, Solukhumbu (Watanabe and Komarek, 1994).

27. Coelosphaerium dubium Grun.: Rice field at Phoksiltar, $400 \mathrm{~m}$, near Sunkoshi river, Udayapur (Rai and Misra, 2010).

28. Cyanobacteria epiphyticum Kom. et Watn.: Sagarmatha National Park, Solukhumbu (Komarek and Watanabe, 1998).

29. Dactylococcopsis acicularis Lemm.: Fish pond at Hetauda, Makawanpur (Sahay et al., 1993).

30. Dactylococcopsis raphidioides Hansg.: A pond at Ankhu Khola, 640 m, Gorkha (Hirano, 1955); Bakeya river (Sahay et al., 1993).

31. Eucapsis himalayensis Watn. et Kom.: Under a cliff with dripping water at Phakdingma, $2700 \mathrm{~m}$ and a shallow lake near Kongma La, 5300 $\mathrm{m}$, Sagarmatha National Park, Solukhumbu (Watanabe and Komarek, 1994); a pond at Mechi Campus, 93 m, Bhadrapur, Jhapa (Rai and Misra, 2010).

32. Eucapsis minuta Fritsch: Haldia river at Chandranigahapur, Rautahat (Sahay et al., 1993).

33. Gloeocapsa aeruginosa (Carm.) Kütz: On moist calcareous rock at Chandragiri, 2000 m, Kathmandu (Watanabe and Komarek, 1988).

34. Gloeocapsa atrata (Turp.) Kütz.: Mingled with mosses on a brick wall at Balaju water garden, $1300 \mathrm{~m}$, 
Kathmandu (Watanabe and Komarek, 1988).

35. Gloeocapsa decorticans (A.Br.) Richter: Wheat fields at Bara, Parsa and Rautahat (Prasad, 1999).

36. Gloeocapsa dermochroa Näg.: On bank by roadside at Nagarjun, 1500 m, Kathmandu (Watanabe and Komarek, 1988).

37. Gloeocapsa montana Kütz:: On moist rocks at Phulchoki, $2000 \mathrm{~m}$, Kavrepalanchok (Watanabe and Komarek, 1988).

38. Gloeocapsa cf. muralis Kütz.: On damp bank by roadside at Godawari, $1400 \mathrm{~m}$, Lalitpur (Watanabe and Komarek, 1988).

39. Gloeocapsa nigrescens Näg.: Bakeya river (Sahay et al., 1993).

40. Gloeocapsa rupestris Kütz:: On moist soil, rice field and Sugarcane field at Birganj, Parsa (Prasad, 1996).

41. Gloeocapsa sanguinea (Ag.) Kütz.: On bank by roadside at Nagarjun, $1500 \mathrm{~m}$, Kathmandu (Watanabe and Komarek, 1988).

42. Gloeocapsopsis ferruginea Kom. et Watn.: Sagarmatha National Park, Solukhumbu (Komarek and Watanabe, 1998).

43. Gloeothece fusco-lutea Näg.: On damp cliff by roadside at Chobhar, $1350 \mathrm{~m}$, Kathmandu (Watanabe and Komarek, 1988).

44. Gloeothece rupestris (Lyngb.) Born.: Wheat fields at Bara, Parsa and Rautahat (Prasad, 1999).

45. Gomphosphaeria aponina Kütz.: A pond at Pisang, $3100 \mathrm{~m}$, Manang; A stream at Tukucha moor, $2600 \mathrm{~m}$, Mustang (Hirano, 1955).

46. Holopedium irregulare Lagerh.: On damp soil at Balaju, $1300 \mathrm{~m}$,
Kathmandu (Shrestha and Manandhar, 1983).

47. Lemmermanniella sp.: Sagarmatha National Park, Solukhumbu (Komarek and Watanabe, 1998).

48. Mantellum himalayense Kom. et Watn.: Sagarmatha National Park, Solukhumbu (Komarek and Watanabe, 1998).

49. Merismopedia elegans A.Br.: Phewa lake, 967 m, Pokhara, Kaski (Nakanishi, 1986); Chandi river at Chandranigahapur, Rautahat, and Rapti river at Hetauda, Makawanpur (Sahay et al., 1993); rice field and on damp soil around a tap at Hongchur, $850 \mathrm{~m}$, Khotang (Rai and Misra, 2010).

50. Merismopedia glauca (Ehr.) Näg.: A pond at Tadikhola, $630 \mathrm{~m}$, Manang (Hirano, 1955); planktonic in lake, Taudaha, Kathmandu (Joshi, 1979); Chandi river at Chandranigahapur, Rautahat and Lamaha river (Sahay et al., 1993); a stream between Chhukung and Amphu, $4770 \mathrm{~m}$, Sagarmatha National Park, Solukhumbu (Watanabe and Komarek, 1994).

51. Merismopedia minima Beck: Chandi river at Chandranigahapur, Rautahat (Sahay et al., 1993).

52. Merismopedia punctata Meyen: A stream at Tukucha moor, $2600 \mathrm{~m}$, Mustang (Hirano, 1955); planktonic in lake Taudaha, Kathmandu (Joshi, 1979); Chandi river at Chandranigahapur, Rautahat and Bakaya river (Sahay et al., 1993); ditches at Narayanghat, Chitwan (Das and Verma, 1996).

53. Merismopedia tenuissima Lemm.: A pond at Ankhu Khola, 640 m, Gorkha 
(Hirano, 1955); lakes in Pokhara valley, Kaski (Nakanishi, 1986).

54. Trochiscia sp.: Shallow ice core from Yala glacier, $5350 \mathrm{~m}$, Langtang region (Yoshimura et al., 1997; 2006).

55. Microcystis aeruginos Kütz: Lakes in Pokhara valley, Kaski (Hickel, 1973a; Nakanishi, 1986); Chhapkaiya pond, Birganj, Parsa (Prasad, 1996); pond at P.G. Campus, $73 \mathrm{~m}$, Biratnagar (Rai and Misra, 2010).

56. Microcystis flos-aquae (Wittr.) Kirchn.: Phewa lake, 967 m, Pokhara, Kaski (Hirano, 1955); planktonic in lake Taudaha as a water bloom, Kathmandu (Joshi, 1979); Chhapkaiya pond, Birganj, Parsa (Prasad, 1996).

57. Microcystis incerta Lemm.: A pond at Hattisar Campus, $511 \mathrm{~m}$, Dharan, Sunsari (Rai and Misra, 2010).

58. Microcystis marginata (Menegh.) Kütz:: Roadside ditches at Mahendranagar, Kanchanpur (Habib, 1997).

59. Microcystis robusta (Clark) Nygaard: Planktonic in lake Taudaha, Kathmandu (Joshi, 1979); Kamal pokhari, 73 m, Sukrabare, Kechana, Morang (Rai and Misra, 2010).

60. Microcystis stagnalis Lemm.: A stream at Tukucha moor, $2600 \mathrm{~m}$, Mustang (Hirano, 1955); Pimbahal pond at Pimbahal, $1300 \mathrm{~m}$, Patan, Lalitpur (Joshi, 1979).

61. Nephrococcus sp.: On moist calcareous rock at Chandragiri, 2000 $\mathrm{m}$, Kathmandu (Watanabe and Komarek, 1988).

62. Synechococcus aeruginosus Näg.: A stream at Tukucha moor, $2600 \mathrm{~m}$, Mustang (Hirano, 1955).
63. Synechococcus elongatus Näg.: Haldia river at Chandranigahapur, Rautahat (Sahay et al., 1993).

64. Synechocystis aquatilis Sauvageau: Chandi river at Chandranigahapur, Rautahat and Kara river at Hetauda, Makawanpur (Sahay et al., 1993); Khageri Khola at Tikauli, Chitwan (Das and Verma, 1996); Madhuban and Kusaha, Sunsari (Jha and Kargupta, 2001).

65. Synechocystis pevalekii Ercegovic: Fish pond at Hetauda, Makawanpur (Sahay et al., 1993); Khageri Khola at Tikauli, Chitwan (Das and Verma, 1996).

66. Woronichinia kuselae Watn. et. Kom.: A shallow lake near Kongma La, 5300 m, Sagarmatha National Park, Solukhumbu (Watanabe and Komarek, 1994); Titrigachi pond, 206 m, Koshi Tappu, Sunsari (Rai and Misra, 2010)

Family: Entophysalidaceae Geitler

67. Chlorogloea cf. microcystoides Geitler: Sagarmatha National Park, Solukhumbu (Komarek and Watanabe, 1998).

68. Chlorogloea simplex Watn. et Kom.: Under a cliff with dripping water at Phakdingma, $2700 \mathrm{~m}, \quad$ SNP, Solukhumbu (Watanabe and Komarek, 1994); Gupha Pokhari lake, 2950 m, Nundhaki, Sankhuwasabha (Rai and Misra, 2010).

69. Entophysalis rubra Watn. et Kom.: A shallow lake near Kongma La, 5300 $\mathrm{m}$; a stream at Dusa, $4530 \mathrm{~m}$, Sagarmatha National Park, Solukhumbu (Watanabe and Komarek, 1994) 
Family: Chamaesiphonaceae Borzi

70. Chamaesiphon confervicola A.Br. ex Rabenh.: A rapid stream of holy bathing water in a small temple at Godawari, $1400 \mathrm{~m}$, Lalitpur (Watanabe and Komarek, 1988).

71. Chamaesiphon incrustans Grun. f. asiatica Will.: A glacier lake at Gosain kunda, $4500 \mathrm{~m}$, Rasuwa (Hirano, 1969).

72. Chamaesiphon cf. jaoi Hällfors (Syn. Ch. Clavatus Jao): Sagarmatha National Park, Solukhumbu (Komarek and Watanabe, 1998).

73. Chamaesiphon cf. minimus Schmidle: Sagarmatha National Park, Solukhumbu (Komarek and Watanabe, 1998).

74. Chamaesiphon minutus Rostaf. ex Lemm.: A rapid stream of holy bathing water in a small temple at Godawari, $1400 \mathrm{~m}$, Lalitpur (Watanabe and Komarek, 1988).

75. Chamaesiphon palssahtiae Kom. et Watn.: Sagarmatha National Park, Solukhumbu (Komarek and Watanabe, 1998).

76. Clastidium nepalense Kom. et Watn.: Sagarmatha National Park, Solukhumbu (Komarek and Watanabe, 1998).

77. Clastidium cf. sicyoideum Li: Sagarmatha National Park, Solukhumbu (Komarek and Watanabe, 1998)

Family: Pleurocapsaceae Geitler

78. Cyanosarcina sp.: Mingled with mosses on a brick wall at Balaju water garden, $1300 \mathrm{~m}$, Kathmandu (Watanabe and Komarek, 1988).

79. Myxosarcina spectabilis Geitler: Periphyton on stones in Sera Khola,
430 m, Panchakanya, Sunsari (Rai and Misra, 2010).

Family: Hyellaceae Borzi

80. Xenococcus luteo-violaceus Kom. et Watn.: Sagarmatha National Park, Solukhumbu (Komarek and Watanabe, 1998).

\section{Family: Oscillatoriaceae Kirchner}

81. Arthrospira gomontiana Setchell: Haripur and Kusaha, Sunsari (Jha and Kargupta, 2001).

82. Arthrospira khannae Drouet et Strickland: Pitchhra pond and canal, $72 \mathrm{~m}$, Biratnagar, Morang (Rai and Misra, 2010).

83. Arthrospira massartii Kuffareth: Haldia river at Chandranigahapur, Rautahat (Sahay et al., 1993).

84. Arthrospira platensis Nordst. ex Gom.: A pond at Nagwa, Birganj, Parsa (Prasad, 1996).

85. Arthrospira spirulinoides Ghose: Municipal drain near Bus stand at Narayanghat, Chitwan (Das and Verma, 1996).

86. Blennothrix ganeshii Watn. et Kom.: Stony substrate in the stream at Godawari, 1400 m, Lalitpur (Watanabe and Komarek, 1988).

87. Hydrocoleus homoeotrichum Kütz.: Stream at Kungbachen, $4000 \mathrm{~m}$ (Hirano, 1984).

88. Leptolyngbya perelegans Lemm. ex Anagn. et Kom. (Syn. Lyngbya perelegans Lemm.): A small pond south of Rara lake, 3030 m, Mugu (Watanabe, 1995).

89. Leptolyngbya rivulariarum Gom. ex Anagn. et Kom. (Syn. Lyngbya rivulariarum Gom.): Submerged rock 
in a streamlet flowing into Rara lake, 2970 m, Mugu (Watanabe, 1995).

90. Lyngbya aerugineo-coerulea Kütz. ex Gom.: Langtang river, $2000 \mathrm{~m}$, Rasuwa (Hirano, 1969); A rapid stream outpouring over a concrete dam at Taudaha, 1350 m, Kathmandu (Watanabe and Komarek, 1988).

91. Lyngbya allorgei Frémy: On damp bank by roadside at Godawari; on brick wall near the gates of fish farm at Godawari, $1400 \mathrm{~m}$, Lalitpur; on moist concrete wall by roadside, $2 \mathrm{~km}$ north of Chabahil, $1300 \mathrm{~m}$, Kathmandu (Watanabe and Komarek, 1988); Sharda river near Mahendranagar, Kanchanpur (Habib, 1997).

92. Lyngbya bipunctata Lemm.: On brick wall near the gates of fish farm at Godawari, $1400 \mathrm{~m}$, Lalitpur (Watanabe and Komarek, 1988).

93. Lyngbya birgei Smith: Sundarijal, Kathmandu; Patan, Lalitpur (Shrestha and Manandhar, 1983); Betana wetland, 123 m, Belbari, Morang (Rai and Misra, 2010).

94. Lyngbya confervoides Ag. ex Gom.: Rice field at Lamjung (Panta and Gupta, 1999).

95. Lyngbya contorta Lemm.: A pond at Indo-Nepal roadside at Mahendranagar, Kanchanpur (Habib, 1997).

96. Lyngbya epiphytica Hieron.: A glacier lake at Langtang Himal, 3700 m, Rasuwa (Hirano, 1969).

97. Lyngbya hieronymusii Lemm.: A pond near Sharda river, Mahendranagar, Kanchanpur (Habib, 1997); pond at Birendra Sabha Griha, $72 \mathrm{~m}$, Biratnagar, Morang (Rai and Misra, 2010).
98. Lyngbya lagerheimii Mob. ex Gom.: A rapid stream outpouring over a concrete dam at Taudaha lake, 1350 m, Kathmandu (Watanabe and Komarek, 1988).

99. Lyngbya lattissima Prescott: Budhi Rapti river at Chitrasari, Chitwan (Das and Verma, 1996).

100. Lyngbya limnetica Lemm.: On a submerged stone in a stream at Walangchung and Kungbachen, 4000 m (Hirano, 1984).

101. Lyngbya maior Menegh.: On moist concrete wall by roadside, $2 \mathrm{~km}$ north of Chabahil, 1300 m, Kathmandu (Watanabe and Komarek, 1988).

102. Lyngbya majuscula Harv. ex Gom.: Betana wetland, $123 \mathrm{~m}$, Belbari, Morang (Rai and Misra, 2010).

103. Lyngbya sordida Zanard. ex Gom.: A stream at Lirum glacier, $3900 \mathrm{~m}$, Rasuwa (Hirano, 1969).

104. Microcoleus chthonoplastes Thuret ex Gom.: Khair Khola at Tandi, Chitwan (Das and Verma, 1996); rice field at Sawane-Thingabari, $500 \mathrm{~m}$, Panchakanya, Sunsari (Rai and Misra, 2010).

105. Microcoleus lacustris Rabenh. ex Farlow: Rice field at Lamjung (Panta and Gupta, 1999); Madhuban, Sunsari (Jha and Kargupta, 2001).

106. Microcoleus sociatus W. West et G.W. West: Damp soil by roadside at Jasbire on the way to Maipokhari, 2010 m, Ilam (Rai and Misra, 2010).

107. Microcoleus subtorrulosus Bréb. ex Gom.: Wheat fields at Bara, Parsa and Rautahat (Prasad, 1999).

108. Microcoleus vaginatus Vauch. ex Gom.: On damp cliff by roadside at Chobhar, 1350 m, Kathmandu (Watanabe and Komarek, 1988). 
109. Oscillatoria acuminata Gom.: A rapid stream outpouring over a concrete dam at Taudaha lake, 1350 $\mathrm{m}$, Kathmandu (Watanabe and Komarek, 1988).

110. Oscillatoria acuta Bruhl et Biswas, orth. mut. Geitler: Haripur, Sunsari (Jha and Kargupta, 2006).

111. Oscillatoria acutissima Kufferath: Khumbu, East Nepal (Kuselfetzmann, 1969); a streamlet connecting fish pond at Godawari, $1400 \mathrm{~m}$, Lalitpur (Watanabe and Komarek, 1988).

112. Oscillatoria agardhii Gom.: A small stream near Langtang Base Camp, 3800 m, Rasuwa (Hirano, 1969); Rapti river at Hetauda, Makawanpur (Sahay et al., 1993); Jayashree khola at Gaindakot, Nawalparasi (Das and Verma, 1996).

113. Oscillatoria amoena Kütz. ex Gom.: Langtang, 2500 m, Rasuwa (Hirano, 1969); on damp wall at Alko Hiti, Patan, Lalitpur (Joshi, 1977); roadside ditches at Mahendranagar, Kanchanpur (Habib, 1997).

114. Oscillatoria amoena (Kütz.) Gom. var. non-granulata Ghose: Dharapani Pandhero at Damku, $1690 \mathrm{~m}$, Khotang (Rai and Misra, 2010).

115. Oscillatoria amphibia Ag. ex Gom.: A pond at Pisang, $3100 \mathrm{~m}$, Manang (Hirano, 1955).

116. Oscillatoria anguina Bory ex Gom.: A rapid stream outpouring over a concrete dam at Taudaha lake, 1350 m, Kathmandu (Watanabe and Komarek, 1988); Malaya roadside ditches, $72 \mathrm{~m}$, Biratnagar, Morang (Rai and Misra, 2010).
117. Oscillatoria animalis Ag. ex Gom.: A glacier lake at Langtang Himal, 3700 m, Rasuwa (Hirano, 1969).

118. Oscillatoria annae van Goor: Rice field at Malangwa, Sarlahi; Kara river at Hetauda, Makawanpur (Sahay et al., 1993).

119. Oscillatoria articulata Gardner: Haldia river at Chandranigahapur, Rautahat (Sahay et al., 1993).

120. Oscillatoria borneti Zukal: Jayashree khola at Gaindakot, Nawalparasi (Das and Verma, 1996).

121. Oscillatoria brevis Kütz. ex Gom.: Langtang river near Langtang Base Camp, 3800 m, Rasuwa (Hirano, 1969).

122. Oscillatoria chalybea Mertens ex Gom.: Haripur, Madhuban and Kusaha, Sunsari (Jha and Kargupta, 2001; 2006).

123. Oscillatoria chlorina Kütz. ex Gom.: Moist soil at Chakupat, Patan, Lalitpur (Joshi, 1977); Haripur and Kusaha, Sunsari (Jha and Kargupta, 2001; 2006).

124. Oscillatoria cortiana Menegh. ex Gom.: Dharapani pandhero at Damku, 1690 m, Khotang (Rai and Misra, 2010).

125. Oscillatoria curviceps Ag. ex Gom.: Rice field at Lamjung (Panta and Gupta, 1999).

126. Oscillatoria deflexa G. West et G.S. West: Kathmandu (Watanabe, 1971); on damp cliff by roadside at Chobhar, 1350 m, Kathmandu (Watanabe and Komarek, 1988).

127. Oscillatoria foreaui Frémy: A pond near Sharda dam, Mahendranagar, Kanchanpur (Habib, 1997).

128. Oscillatoria formosa Bory ex Gom.: A pond at Arughat Bazaar, $640 \mathrm{~m}$ and 
Luitel Bhanjyang, $770 \mathrm{~m}$, Gorkha (Hirano, 1955); on water-logged soil at Patan, Lalitpur and drainage soil at Sundarijal, Kathmandu (Joshi, 1977); water logged soil, Lamosangu, Sindhupalchok (Joshi, 1979); Bagmati river at Karmaiya (Sahay et al., 1993); Kaparphori ditches at Tikauli, Chitwan (Das and Verma, 1996); Ghariyar pond, rice, wheat and sugarcane fields, Birganj, Parsa (Prasad, 1996); rice fields at Sardanagar, Rampur, Chitwan (Prasad and Prasad, 2001).

129. Oscillatoria granulata Gardner: A shallow stagnant water, $3 \mathrm{~km}$ north of Chabahil, 1300 m, Kathmandu (Watanabe and Komarek, 1988).

130. Oscillatoria hamelii Frémy: Haripur and Kusaha, Sunsari (Jha and Kargupta, 2001; 2006).

131. Oscillatoria irrigua Kütz. ex Gom.: A stream at Kali Gandaki, $2540 \mathrm{~m}$, Mustang (Hirano, 1955); Khageri Khola at Tikauli, Chitwan (Das and Verma, 1996); Bhandara, Chitwan (Prasad and Prasad, 2001).

132. Oscillatoria jasorvensis Vouk.: Moist soil at Chakupat, $1300 \mathrm{~m}$, Lalitpur; near Pashupatinath, $1300 \mathrm{~m}$, Kathmandu (Joshi, 1977); on wall of hot spring water at Tatopani, $700 \mathrm{~m}$, Sindhupalchok (Joshi, 1979).

133. Oscillatoria laete-virens Crouan ex Gom.: Rice field at Lamjung (Panta and Gupta, 1999).

134. Oscillatoria limosa Ag. ex Gom.: On soil of hot spring water at Tatopani, 700 m, Sindhupalchok (Joshi, 1979); Kathmandu (Shrestha and Manandhar, 1983); Chandi river at Chandranigahapur, Rautahat and Kara river at Hetauda, Makawanpur (Sahay et al., 1993); main dam of Khageri Khola at Tikauli, Chitwan (Das and Verma, 1996); Madhuban, Haripur and Kusaha, Sunsari (Jha and Kargupta, 2001; 2006); rice fields at Sardanagar, Rampur, Chitwan; rice field at Khumaltar, 1300 m, Lalitpur; rice fields at Thimi, Bhaktapur (Prasad and Prasad, 2001); Naharchowk ditches near Satasidham river, $128 \mathrm{~m}$, Shivaganj, Jhapa (Rai and Misra, 2010).

135. Oscillatoria mougeotii Kütz.: Rice field at Malangwa, Sarlahi (Sahay et al., 1993).

136. Oscillatoria nigra Vaucher: Fish pond at Hetauda, Makawanpur (Sahay et al., 1993).

137. Oscillatoria obscura Bruhl et Biswas: On water logged soil, Lamosangu, Sindhupalchok (Joshi, 1979); Haripur, Sunsari (Jha and Kargupta, 2006).

138. Oscillatoria okeni Ag. ex Gom. var. gracilis Kütz.: Kathmandu (Watanabe, 1971); on damp cliff by roadside at Chobhar, $1350 \mathrm{~m}$; a small pond at Bajrabarahi, $1300 \mathrm{~m}$; a rapid stream outpouring over a concrete dam at Taudaha lake, $1350 \mathrm{~m}$, Kathmandu (Watanabe and Komarek, 1988).

139. Oscillatoria ornata Kütz. ex Gom.: Haripur, Sunsari (Jha and Kargupta, 2006).

140. Oscillatoria princeps Vauch. ex Gom.: A pond at Patan Dhoka, 1300 m, Lalitpur (Hirano, 1963); Narayani river side at Narayanghat, Chitwan (Upadhyaya, 1979); Jayashree khola at Gaindakot, Nawalparasi; Kara river at Hetauda, Makawanpur; Khageri Khola at Tikauli, Chitwan (Das and Verma, 1996); rice and sugarcan 
fields, Birganj, Parsa (Prasad, 1996); roadside ditches at Mahendranagar, Kanchanpur (Habib, 1997); Madhuban, Haripur and Kusaha, Sunsari (Jha and Kargupta, 2001; 2006); Malaya roadside ditches, $72 \mathrm{~m}$, Biratnagar, Morang (Rai and Misra, 2010).

141. Oscillatoria proboscidea Gom.: Hot spring of Tatopani on the way to jomsom, $\quad 1300 \mathrm{~m}, \quad$ Mustang (Upadhyaya, 1979); on damp cliff by roadside at Chobhar, $1350 \mathrm{~m}$, Kathmandu (Watanabe and Komarek, 1988); on moist soil at Birganj (Prasad, 1996); roadside ditches at Mahendranagar, Kanchanpur (Habib, 1997); Sarochia pond, $72 \mathrm{~m}$, Biratnagar, Morang (Rai and Misra, 2010).

142. Oscillatoria cf. proteus Skuja: On moist concrete wall by roadside, $2 \mathrm{~km}$ north of Chabahil, $1300 \mathrm{~m}$, Kathmandu (Watanabe and Komarek, 1988).

143. Oscillatoria pseudominima Skuja: A glacier lake at Langtang Himal, 3700 m, Rasuwa (Hirano, 1969).

144. Oscillatoria sancta Kütz. ex Gom.: Kaparphori ditches at Tikauli, Chitwan (Das and Verma, 1996); Madhuban, Haripur and Kusaha, Sunsari (Jha and Kargupta, 2001; 2006); Raja Rani lake, $500 \mathrm{~m}$, Bhogateni, Morang (Rai and Misra, 2010).

145. Oscillatoria splendida Grev. ex Gom.: A stream at Lirum glacier, 3900 m, Rasuwa (Hirano, 1969); Chimdi lake, 73 m, Sunsari (Rai and Misra, 2010).

146. Oscillatoria subbrevis Schmidle: On moist soil at Patan Dhoka, 1300 m,
Lalitpur (Joshi, 1977); on wall of hot spring water, Tatopani, Sindhupalchok (Joshi,1979); Kara river at Hetauda, Makawanpur and rice field at Malangwa, Sarlahi (Sahay et al., 1993).

147. Oscillatoria subproboscidea $\mathrm{G}$. West et G.S. West: On damp cliff by roadside at Chobhar, $1350 \mathrm{~m}$, Kathmandu (Watanabe and Komarek, 1988).

148. Oscillatoria terebriformis Ag.: A pond at Thaple Himal, $4000 \mathrm{~m}$, Manang (Hirano, 1955).

149. Oscillatoria vizagapatensis Rao: Haripur, Sunsari (Jha and Kargupta, 2006).

150. Phormidium ambiguum Gom.: A pond at Boudhanath, $1300 \mathrm{~m}$, Kathmandu (Hirano, 1963); rice fields at Kathmandu (Baral et al., 1988); on damp soil around a tap water, $2 \mathrm{~km}$ below Makpa, 1440 m, Khotang (Rai and Misra, 2010).

151. Phormidium anomala Rao: A fish pond at Mahendranagar, Kanchanpur (Habib, 1997).

152. Phormidium autumnale Ag. ex Gom.: A pond at Chittrey pass, 2660 $\mathrm{m}$, Manang (Hirano, 1955); Thimi, Bhaktapur (Shrestha and Manandhar, 1983).

153. Phormidium corium Ag. ex Gom.: A pond at Patan Dhoka, $1300 \mathrm{~m}$, Lalitpur (Hirano, 1963); on damp bank by roadside at Godawari, 1400 m, Lalitpur (Watanabe and Komarek, 1988).

154. Phormidium favosum Bory ex Gom.: A stream at Mewa valley, $4150 \mathrm{~m}$ (Hirano, 1984). 
155. Phormidium fragile Menegh. ex Gom.: Rani pokhari, $1300 \mathrm{~m}$, Kathmandu (Hirano, 1963).

156. Phormidium incrustatum Näg. ex Gom.: A fish pond at Mahendranagar, Kanchanpur (Habib, 1997).

157. Phormidium inundatum Kütz. ex Gom.: A stream at Mewa valley, 4150 m (Hirano, 1984).

158. Phormidium jadinianum Gom.: Rice field at Lamjung (Panta and Gupta, 1999).

159. Phormidium laminnatum Ag. ex Gom.: Fresh water at Kaligandaki, $2500 \mathrm{~m}$, Mustang; a stream at Tukucha moor, $2600 \mathrm{~m}$, Mustang (Hirano, 1955).

160. Phormidium laminosum Gom.: A stream at Mewa valley, $4150 \mathrm{~m}$ (Hirano, 1984).

161. Phormidium lucidum Kütz. ex Gom.: Sirsiya river and moist soils at Birganj, Parsa (Prasad, 1996); wheat fields at Bara, Parsa and Rautahat (Prasad, 1999).

162. Phormidium molle Kütz. ex Gom.: Madhuban and Haripur, Sunsari (Jha and Kargupta, 2001).

163. Phormidium papyraceum Ag. ex Gom.: A glacier lake at Rarkya pass, 4850 m, Manang (Hirano, 1963?).

164. Phormidium purpurascens Kütz. ex Gom.: A stream at Pisang, $3100 \mathrm{~m}$, Manang (Hirano, 1955).

165. Phormidium retzii Ag. ex Gom.: Kathmandu (Watanabe, 1971); a stream at Mewa valley, $4150 \mathrm{~m}$ (Hirano, 1984); on damp cliff by roadside at Chobhar, $1350 \mathrm{~m}$, Kathmandu (Watanabe and Komarek, 1988).
166. Phormidium rubroterricola Gardner: Haripur and Kusaha, Sunsari (Jha and Kargupta, 2001).

167. Phormidium stagnina Rao: Rice field at Lamjung (Panta and Gupta, 1999).

168. Phormidium subfuscum Kütz. ex Gom.: Roadside drains at Ilam Bazaar, 1208 m, Ilam (Rai and Misra, 2010).

169. Phormidium tenue (Ag. ex Gom.) Anagn. et Kom. (Syn. Oscillatoria tenuis Ag. ex Gom.): A small pond south of Rara lake, $3030 \mathrm{~m}$, Mugu (Watanabe, 1995); Rapti river at Hetauda, Makawanpur (Sahay et al., 1993).

170. Phormidium uncinatum Ag. ex Gom.: Kathmandu (Watanabe, 1971); on damp bank by roadside at Godawari, $1400 \mathrm{~m}$, Lalitpur (Watanabe and Komarek, 1988).

171. Phormidium usterii Schmidle: A fish pond at Mahendranagar, Kanchanpur (Habib, 1997).

172. Phormidium valderiae Schmidle: A small stream near Langtang Base Camp, 3800 m, Rasuwa (Hirano, 1969).

173. Schizothrix flammea Kom. et Watn.: Sagarmatha National Park, Solukhumbu (Komarek and Watanabe, 1998).

174. Schizothrix friesii Ag. ex Gom.: On banks by roadside at Nagarjun, 1500 $\mathrm{m}$, Kathmandu (Watanabe and Komarek, 1988).

175. Schizothrix heufleri Grun.: On moist calcareous rock at Chandragiri, 2000 m, Kathmandu (Watanabe and Komarek, 1988).

176. Schizothrix lacustris A.Br. ex Gom.: A glacier lake at Langtang Himal, 3700 m, Rasuwa (Hirano, 1969). 
177. Schizothrix pulvinata Kütz. ex Gom.: Rice fields at Kathmandu (Baral et al., 1988).

178. Schizothrix purpurascens Kütz. ex Gom.: Mingled with mosses at Nagarkot, 1600 m, Bhaktapur (Watanabe and Komarek, 1988).

179. Schizothrix radius-solis Watn. et Kom.: A small pond north west of Pangpoche, $4550 \mathrm{~m}$, SNP, Solukhumbu (Watanabe and Komarek, 1994).

180. Spirulina gigantea Schmidle: Rupa and Khaste lakes, $900 \mathrm{~m}$, Pokhara, Kaski (Hickel, 1973a); fish pond at Hetauda, Makawanpur (Sahay et al., 1993); Jayashree khola at Gaindakot, Nawalparasi (Das and Verma, 1996); Haripur and Kusaha, Sunsari (Jha and Kargupta, 2001).

181. Spirulina major Kütz. ex Gom.: Bagmati river at Karmaiya and rice field at Malangwa, Sarlahi (Sahay et al., 1993); Rawa Khola, 720 m, Manglabare, Khotang (Rai and Misra, 2010).

182. Spirulina meneghiniana Zanard. ex Gom.: Fish pond and Kara river at Hetauda, Makawanpur (Sahay et al., 1993); Madhuban and Kusaha, Sunsari (Jha and Kargupta, 2001).

183. Spirulina princeps W. West et G.S. West: Fish pond at Hetauda, Makawanpur (Sahay et al., 1993); Madhuban, Haripur and Kusaha, Sunsari (Jha and Kargupta, 2001); Kamal pokhari, 73 m, Sukrabare, Kechana, Morang (Rai and Misra, 2010).

184. Spirulina subsalsa Oerst. ex Gom.: Bagmati river at Karmaiya and Rice field at Malangwa, Sarlahi (Sahay et al., 1993); Madhuban and Kusaha,
Sunsari (Jha and Kargupta, 2001); Pitchhra pond and canal, $72 \mathrm{~m}$, Biratnagar, Morang (Rai and Misra, 2010).

185. Spirulina subtilissima Kütz. ex Gom.: Madhuban and Kusaha, Sunsari (Jha and Kargupta, 2001).

186. Symploca flotowiana Kütz.: On moist concrete wall by roadside, $2 \mathrm{~km}$ north of Chabahil, 1300 m, Kathmandu (Watanabe and Komarek, 1988).

Family: Nostocaceae Kütz.

187. Anabaena augstumalis var. marchica Lemm.: A ditch at Jayashree khola at Gaindakot, Nawalparasi (Das and Verma, 1996).

188. Anabaena azollae: Rice field at Birganj, Parsa (Prasad, 1996).

189. Anabaena circinalis Rabenh. ex Born. et Flah.: Rupa lake, 967 m, Pokhara, Kaski (Nakanishi, 1986).

190. Anabaena doliolum Bhardwaja: Rice fields at Kathmandu (Baral et al., 1988); rice field at Birganj, Parsa (Prasad, 1996).

191. Anabaena fertilissima Rao: Rice fields at Kathmandu (Baral et al., 1988).

192. Anabaena flos-aquae (Lyngb.) Bréb. ex Born. et Flah.: Rice field at Lamjung (Panta and Gupta, 1999).

193. Anabaena globosa Hirano: Rani pokhari, 1300 m, Kathmandu (Hirano, 1963).

194. Anabaena iyengarii Bharadwaja: Chandi river at Chandranigahapur, Rautahat (Sahay et al., 1993); a pond near Sharda dam, Mahendranagar, Kanchanpur (Habib, 1997); Titrigachi pond, 206 m, Koshi Tappu, Sunsari (Rai and Misra, 2010). 
195. Anabaena iyengarii Bharadwaja var. tenuis Rao: Haripur and Kusaha, Sunsari (Jha and Kargupta, 2001); rice field at Phoksiltar, $400 \mathrm{~m}$, near Sunkoshi river, Udayapur (Rai and Misra, 2010).

196. Anabaena lapponica Borge: A small pond south of Rara lake, 3030 m, Mugu (Watanabe, 1995).

197. Anabaena cf. laxa (Rabenh.) A.Br. in Born. et Flah.: Under a cliff with dripping water at Phakdingma, 2700 $\mathrm{m}$; a shallow streamlet north east of Chhukung, $4770 \mathrm{~m}$; a shallow lake near Kongma La, 5300 m, Sagarmatha National Park, Solukhumbu (Watanabe and Komarek, 1994).

198. Anabaena naviculoides Fritsch: Haripur and Kusaha, Sunsari (Jha and Kargupta, 2001).

199. Anabaena oscillarioides Bory ex Born. et Flah.: A small pond south of Rara lake, 3030 m, Mugu (Watanabe, 1995).

200. Anabaena papillosa Hirano: A branch stream of Langtang river, 2800 m, Rasuwa (Hirano, 1969).

201. Anabaena sphaerica Born. et Flah. var. tenuis G.S. West: Khageri Tal at Tikauli, Chitwan (Das and Verma, 1996).

202. Anabaena unispora Gardner: A ditch along by-pass road at Gaindakot, Nawalparasi (Das and Verma, 1996).

203. Anabaena variabilis Kütz. ex Born. et Flah.: A ditch along by-pass road at Gaindakot, Nawalparasi (Das and Verma, 1996); rice field at Khumaltar, 1300 m, Lalitpur; rice field at Thimi, Bhaktapur (Prasad and Prasad, 2001).

204. Anabaena volzii Lemm.: A puddle along Khair Khola at Belchi, Tandi,
Chitwan (Das and Verma, 1996); rice field at Phoksiltar, $400 \mathrm{~m}$, near Sunkoshi river, Udayapur (Rai and Misra, 2010).

205. Anabaenopsis raciborskii Wolosz: Chhapkaiya pond, Birganj, Parsa (Prasad, 1996).

206. Aulosira fertilissima Ghose: Nepal (Singh, 1961); Municipal drain near Bus stand at Narayanghat, Chitwan (Das and Verma, 1996); rice field at Jagannathpur, Parsa; rice field at Parwanipur, Bara (Prasad and Prasad, 2001).

207. Aulosira fertilissima Ghose var. tenuis Rao: A pond near Indo-Nepal border, Mahendranagar, Kanchanpur (Habib, 1997).

208. Aulosira fritschii Bharadwaja: Khageri Tal at Tikauli, Chitwan (Das and Verma, 1996).

209. Aulosira implexa Born. et Flah.: Suryabinayak, 1300 m, Bhaktapur; Godawari pond, 1300 m, Lalitpur (Shrestha and Manandhar, 1983).

210. Aulosira prolifica Bharadwaja: Khair Khola at Tandi, Chitwan (Das and Verma, 1996).

211. Cylindrospermum cf. breve Welsh: Epiphytic on Scytonema among wet mosses on rocky substrate and on roots of trees in streaming and dripping water at Phakdingma, 2700 m, Sagarmatha National Park, Solukhumbu (Watanabhe and Komarek, 1994).

212. Cylindrospermum indicum Rao: Rocky substrate overflowed by spring water between Lardze and Namche Bazaar, 3100 m, Sagarmatha National Park, Solukhumbu (Watanabe and Komarek, 1994). 
213. Cylindrospermum majus Kütz. ex Born. et Flah.: Chandi river at Chandranigahapur, Rautahat (Sahay et al., 1993); Suryabinayak, $1300 \mathrm{~m}$, Bhaktapur (Shrestha and Manandhar, 1983).

214. Cylindrospermum minutissimum Collins: On damp cliff by roadside at Chobhar, $1350 \mathrm{~m}$, Kathmandu (Watanabe and Komarek, 1988); a small pond south of Rara lake, 3030 m, Mugu (Watanabe, 1995).

215. Cylindrospermum stagnale Kütz. ex Born. et Flah.: In stagnant water at Sanegaun, Patan, Lalitpur (Joshi, 1979); gelatinous aggregation in mosses at Nagarkot, $1600 \mathrm{~m}$, Bhaktapur; on damp bank by roadside at Godawari, $1400 \mathrm{~m}$, Lalitpur (Watanabe and Komarek, 1988); rice fields at Kathmandu (Baral et al., 1988); a pond at Mahendranagar, Kanchanpur (Habib, 1997).

216. Cylindrospermum stagnale Kütz. ex Born. et Flah. forma variabilis Prasad: Chimdi lake, 73 m, Sunsari (Rai and Misra, 2010).

217. Nodularia harveyana (Thw. in Harv.) Thur. ex Born. et Flah.: Kathmandu (Watanabe, 1971); shallow water near west shore of Rara lake, 2970 m, Mugu (Watanabe, 1995).

218. Nostoc calcicola Bréb. ex Born. et Flah.: On damp bank by roadside at Godawari, 1400 m, Lalitpur (Watanabe and Komarek, 1988); rice fields at Kathmandu (Baral et al., 1988).

219. Nostoc commune Vauch. ex Born. et Flah.: Water tank, rice field and sugarcan fields at Birganj, Parsa (Prasad, 1996); rice fields at Parwanipur, Bara (Prasad and Prasad,
2001); Maipokhari lake, 2150 m, Ilam

(Rai and Misra, 2010).

220. Nostoc ellipsosporum (Desm.) Rabenh. ex Born. et Flah.: On moist soil at Senegaun, Patan, Lalitpur (Joshi, 1979); on damp cliff by roadside at Chobhar, $1350 \mathrm{~m}$, Kathmandu; on damp bank by roadside at Godawari, $1400 \mathrm{~m}$, Lalitpur (Watanabe and Komarek, 1988); rice fields at Kathmandu (Baral et al., 1988).

221. Nostoc linckia (Roth.) Bornet ex Born. et Flah.: Godawari, $1400 \mathrm{~m}$, Lalitpur; Thimi, Bhaktapur (Shrestha and Manandhar, 1983); a pond at Mahendranagar, Kanchanpur (Habib, 1997); rice fields at Sardanagar, Rampur, Chitwan; rice fields at Parwanipur, Bara; rice field at Khumaltar, 1300 m, Lalitpur; rice fields at Panga, Kirtipur, Kathmandu (Prasad and Prasad, 2001).

222. Nostoc microscopicum Carm. ex Born. et Flah.: Wheat fields at Bara, Parsa and Rautahat (Prasad, 1999).

223. Nostoc muscorum Ag. ex Born. et Flah.: On water logged soil in rice field at Senegaun, Patan, Lalitpur (Joshi, 1979).

224. Nostoc paludosum Kütz. ex Born. et Flah.: A pond at Patan Dhoka, 1300 m, Lalitpur (Hirano, 1963).

225. Nostoc sphericum Vauch. ex Born. et Flah.: Rice fields at Thimi, Bhaktapur (Prasad and Prasad, 2001).

226. Nostoc spongiaeforme Ag. ex Born. et Flah.: On moist concrete wall by roadside, $2 \mathrm{~km}$ north of Chabahil, 1300 m, Kathmandu (Watanabe and Komarek, 1988); rice fields at Tamnagar, Belbas and Devinagar at Butawal (Gyawali and Prasad, 1999). 
227. Pseudanabaena catenata Lauterb.: On damp cliff by roadside at Chobhar, $1350 \mathrm{~m}$; Taudaha lake, $1350 \mathrm{~m}$, Kathmandu (Watanabe and Komarek, 1988).

228. Pseudanabaena mucicola (Naum. et Huber-Pest.) Bourr. Emend. Chang, Koest et Wanner (Syn. Phormidium mucicola Naum. et Huber-Pest.): Submerged rock in a streamlet flowing into Rara lake, 2970 m, Mugu (Watanabe, 1995).

229. Arthronema africanum (Schwabe et Simonsen) Komárek et Lukavský: High plateaus in Nepal (Komárek and Lukavský, 1988).

230. Raphidiopsis curvata Fritsch et Rich.: Sirsiya river and seawage site at Birganj, Parsa (Prasad, 1996)

Family: Scytonemataceae Rabenhorst

231. Scytonema arcangelii Born. et Flah.: Thankot, 1300 m, Kathmandu (Shrestha and Manandhar, 1983).

232. Scytonema bewsii Fritsch et Rich.: On damp bank by roadside at Godawari, $1400 \mathrm{~m}$, Lalitpur; gelatinous aggregation in mosses at Nagarkot, $1600 \mathrm{~m}$, Bhaktapur (Watanabe and Komarek, 1988).

233. Scytonema burmanicum Skuja: Sabha Pokhari lake, $4100 \mathrm{~m}$, Sankhuwasabha (Rai and Misra, 2010).

234. Scytonema contorta Watn. et Kom.: On bank by roadside at Nagarjun, $1500 \mathrm{~m}$, Kathmandu (Watanabe and Komarek, 1988).

235. Scytonema fertilia Watn. et Kom.: Mingled with mosses at Nagarkot, 1600 m, Bhaktapur (Watanabe and Komarek, 1988).
236. Scytonema javanicum (Kütz.) Born. ex Born. et Flah.: Roadside ditches at Itahari, $120 \mathrm{~m}$, Sunsari (Rai and Misra, 2010).

237. Scytonema mirabile Dillw. ex Born.: A glacier lake at Langtang Himal, 3700 m, Rasuwa (Hirano, 1969).

238. Scytonema myochrous (Dillw.) Ag. ex Born. et Flah.: Khumbu, East Nepal (Kusel-fetzmann, 1969); on moist calcareous rock at Chandragiri, 2000 m, Kathmandu (Watanabe and Komarek, 1988).

239. Scytonema ocellatum Lyngb. ex Born. et Flah.: On rocks by roadside at Swayambhu, $1300 \mathrm{~m}$, Kathmandu; on brick wall near the gates of fish farm at Godawari, $1400 \mathrm{~m}$, Lalitpur (Watanabe and Komarek, 1988).

240. Scytonema schmidtii Gom.: On brick wall by roadside at Nagarjun, $1500 \mathrm{~m}$, Kathmandu (Watanabe and Komarek, 1988).

241. Scytonema stuposum (Kütz.) Born. ex Born. et Flah.: Epiphytic on bark of Accasia auriculiformis at Botany Department, P.G. Campus, 72 m, Biratnagar, Morang (Rai and Misra, 2010).

242. Tolypothrix cf. arenophila G. West et G.S. West: Mingled with mosses on a brick wall at Balaju water garden, 1300 m, Kathmandu (Watanabe and Komarek, 1988).

243. Tolypothrix distorta Kütz. ex Born. et Flah.: A small pond south of Rara lake, $3030 \mathrm{~m}$ and floating on Rara lake, $2970 \mathrm{~m}$, Mugu (Watanabe, 1995); Mechi pond at Maheshpur, 80 $\mathrm{m}$, Bhadrapur, Jhapa (Rai and Misra, 2010).

244. Tolypothrix phyllophila G. West et G.S. West: On moist calcareous rocks 
at Chandragiri, $2000 \mathrm{~m}$, Kathmandu (Watanabe and Komarek, 1988)

Family: Microchaetaceae Limm.

245. Coleodesmium sagarmathae Kom. et Watn.: Periphyton on stones at streams of Imja river south from Lhotse Shar, $4770 \mathrm{~m}$; outlet of lake near Longponga, south from Ngozumpa glacier, $4650 \mathrm{~m}$; shallow pool east from Kongma La, south west from Nuptse, $5250 \mathrm{~m}$; shallow lake north west from Pangpoche, 4550 m; outlet from Saraswati Kunda, Gosainkund area, Solukhumbu (Komarek and Watanabe, 1990?).

246. Fortiea sp.: Under a cliff with dripping water at Phakdingma, 2700 $\mathrm{m}$, Solukhumbu (Watanabe and Komarek, 1994); Sagarmatha National Park, Solukhumbu (Komarek and Watanabe, 1998).

247. Microchaete cf. aequalis Frémy ex Desikachary: Epiphytic on mosses and liverworts in droping water and on wetted rock and free foots of trees at Phakdingma, $2700 \mathrm{~m}$, Sagarmatha National Park, Solukhumbu (Watanabe and Komarek, 1994).

248. Microchaete tenera Thuret ex Born. et Flah.: A small pond south of Rara lake, $3030 \mathrm{~m}$, Mugu (Watanabe, 1995).

Family: Rivulariaceae Rabenhorst

249. Calothrix atricha Frémy: A ditch along by-pass road at Gaindakot, Nawalparasi (Das and Verma, 1996).

250. Calothrix castellii Massal. ex Born. et Flah.: A ditch along Jayashree khola at Gaindakot, Nawalparasi (Das and Verma, 1996).
251. Calothrix castellii Massal. ex Born. et Flah. var. somastipurense Rao: Epiphytic on decaying leaves of Euphorbia pulcherrima, submerged in ditches at Biratnagar, $72 \mathrm{~m}$, Morang (Rai and Misra, 2010).

252. Calothrix fusca Kütz. ex Born. et Flah.: On damp cliff by roadside at Chobhar, $1350 \mathrm{~m}$, Kathmandu (Watanabe and Komarek, 1988); a fish pond at Mahendranagar, Kanchanpur (Habib, 1997).

253. Calothrix fusca Kütz. ex Born. et Flah. var. crassa Rao: In the mucilage of Anabaena sp. in moist rock at Sundarijal Mai, Sundarijal, Kathmandu (Joshi, 1979); Siddhapokhari, Bhaktapur (Shrestha and Manandhar, 1983).

254. Calothrix cf. gloeocola Skuja: On a dead tree in the south shore of Rara lake; on gelatinous mass of Rivularia cf. hansgirgii on submerged rock in streamlet flowing into Rara lake, 2970 m, Mugu (Watanabe, 1995).

255. Calothrix parietina Thuret ex Born. et Flah.: A stream at Pisang, $3100 \mathrm{~m}$, Manang (Hirano, 1955); Kathmandu (Watanabe, 1971); Patan, Lalitpur (Joshi, 1979); on damp cliff by roadside at Chobhar, $1350 \mathrm{~m}$, Kathmandu (Watanabe and Komarek, 1988).

256. Calothrix scytonemicola Tilden: On damp cliff by roadside at Chobhar, $1350 \mathrm{~m}$, Kathmandu (Watanabe and Komarek, 1988).

257. Gloeotrichia echinulata J.E. Smith ex Rich. var. berhampurense Rao: Mawa river, $230 \mathrm{~m}$, Morang; rice field at Phoksiltar, $400 \mathrm{~m}$, near Sunkoshi river, Udayapur (Rai and Misra, 2010). 
258. Gloeotrichia intermedia Lemm. ex Geitl.: On moist soil, rice field and sugarcan field at Birganj, Parsa (Prasad, 1996); Haripur, Sunsari (Jha and Kargupta, 2001).

259. Gloeotrichia intermedia Lemm. ex Geitl. var. kanwaensis Rao: Mawa river, $230 \mathrm{~m}$, Morang (Rai and Misra, 2010).

260. Gloeotrichia natans Rabenh. ex Born. et Flah.: Lamaha river (Sahay et al., 1993); a puddle at Mahendranagar Bazaar, Kanchanpur (Habib, 1997).

261. Gloeotrichia raciborskii Wolosz. var. kashiense Rao: Chimdi lake, $73 \mathrm{~m}$, Sunsari (Rai and Misra, 2010).

262. Homoeothrix cf. janthina (Born. et Flah.) Starmach: On damp bank by roadside at Godawari, $1400 \mathrm{~m}$, Lalitpur (Watanabe and Komarek, 1988).

263. Rivularia aquatica De Wilde.: Haripur, Sunsari (Jha and Kargupta, 2001).

264. Rivularia hansgirgi Schmidle: On submerged rock in a streamlet flowing into Rara lake, $2970 \mathrm{~m}$, Mugu (Watanabe, 1995); Haripur, Sunsari (Jha and Kargupta, 2001).

265. Rivularia minutula Kütz. ex Born. et Flah.: Nagarjun, 1300 m, Kathmandu (Shrestha and Manandhar, 1983); Mechi pond at Maheshpur, $80 \mathrm{~m}$, Bhadrapur, Jhapa (Rai and Misra, 2010)

Family: Nostochopsidaceae Geitler

266 Nostochopsis lobatus Wood em. Geitl.: A streamlet connecting fish pond at Godawari, $1400 \mathrm{~m}$, Lalitpur (Watanabe and Komarek, 1988); a pond at Godawari, Lalitpur (Shrestha and Manandhar, 1983); on wall of outlet canal at Betana wetland, $123 \mathrm{~m}$, Belbari, Morang (Rai and Misra, 2010).

Family: Stigonemataceae Kirchner

267 Fischerella epiphytica Ghose: Epiphytic on decaying leaves of Euphorbia pulcherrima, submerged in ditches at Biratnagar, $72 \mathrm{~m}$, Morang (Rai and Misra, 2010).

268 Hapalosiphon fontinalis Ag. ex Born.: A small pond south of Rara lake, $3030 \mathrm{~m}$, Mugu (Watanabe, 1995); Maipokhari lake, 2150 m, Ilam (Rai and Misra, 2010).

269 Hapalosiphon cf. intricatus G. West et G.S. West: Gelatinous aggregation with mosses at Nagarkot, $1600 \mathrm{~m}$, Bhaktapur (Watanabe and Komarek, 1988).

270 Hapalosiphon welwitschii G. West et G.S. West: Bagmati river at Karmaiya (Sahay et al., 1993).

271 Stigonema hormoides Kütz. ex Born. et Flah.: Gelatinous aggregation with mosses at Nagarkot, $1600 \mathrm{~m}$, Bhaktapur (Watanabe and Komarek, 1988).

272 Stigonema mamillosum (Lyngb.) Ag. ex Born. et Flah.: A glacier lake at Langtang Himal, $3700 \mathrm{~m}$, Rasuwa (Hirano, 1969); Gokyu lake III, 4770 $\mathrm{m}$, Khumjung, Solukhumbu (Rai and Misra, 2010).

273 Stigonema ocellatum (Dillw.) Thuret ex Born. et Flah.: A glacier lake at Langtang Himal, 3700 m, Rasuwa (Hirano, 1969); Khumbu, East Nepal (Kusel-fetzmann, 1969); a small pond south of Rara lake, $3030 \mathrm{~m}$, Mugu (Watanabe, 1995); Sabha Pokhari lake, $4100 \mathrm{~m}$, Sankhuwasabha (Rai and Misra, 2010). 
S.K. Rai, R.K. Rai and S. Jha / Our Nature (2010) 8: 336-354

274 Westiellopsis sp.: Rice fields at Chandranigahapur, Rautahat; rice field at Khumaltar, 1300 m, Lalitpur; rice fields at Panga, Kirtipur, Kathmandu; rice fields at Thimi, Bhaktapur (Prasad and Prasad, 2001)

\section{Acknowledgements}

Auther are very thankful to Dr. Shiro Koshima, Tokyo Institute of Technology, Ookayama Meguro-Ku, Tokyo, Japan; Dr. Yoshitaka Yoshimura, Faculty of Agriculture, Tamagawa University, Machida, Tokyo, Japan and Dr. V.N. Prasad, Department of Botany, Thakur Ram Multiple Campus, Birgunj for providing the literature on algae of Nepal. Thanks are also due to the Heads of Library, NBRI, CSIR, Lucknow, India and Central Library, TU, Kirtipur, Nepal for the library facilities.

\section{References}

Baral, S.R., D.K. Mishra and H.D. Kumar 1988. In situ nitrogen fixation rates in ten rice fields of Kathmandu valley, Nepal. In Biofertilizers: Potentialities and Problems (Eds. S.P. Sen and P. Palit). Naya Prakash, Calcutta, India. pp. 103-107.

Das, S.N. and B.N. Verma 1996. Algal flroa of Chitwan and Nawalparasi districts of Nepal. Phykos 35(1-2): 119-127.

Desikachary, T.V. 1959. Cyanophyta. I.C.A.R. monograph on algae, New Delhi. 686 p.

Habib, I. 1997. Algal flora from Mahendranagar, Nepal. J .Econ. and Taxon. Bot. (India) 21(1): 19-26.

Hickel, B. 1973a. Limnological investigations in lakes of Pokhara valley, Nepal. Int. Rev. ges Hydrobiol. 58(5): 659-672.

Hickel, B. 1973b. Phytoplanktons in two ponds in Kathmandu valley, Nepal. Int. Rev. ges Hydrobiol. 58(6): 835-842.

Hirano, M. 1955. Fresh water algae. In Fauna and flora of Nepal Himalaya (Ed. H. Kihara). Fauna and Flora Research Society, Kyoto University, Kyoto, Japan. pp. 5-42.

Hirano, M. 1963. Fresh water algae from the Nepal Himalaya, collected by a member of the Japanese Climbing Expedition. Contr. Biol. Lab., Kyoto Univ., Japan. 16: 1-23.

Hirano, M. 1969. Fresh water algae from Langtang Himal, Nepal Himalaya. Contr. Biol. Lab., Kyoto Univ., Japan. 22: 1-42.

Jha, S. and A.N. Kargupta 2001. Cyanobacterial flora of Eastern Koshi basin, Nepal. Ecoprint 8(1): $37-43$.

Jha, S. and A.N. Kargupta 2006. Taxonomy of the genus Oscillatoria Vaucher from the river Koshi basin. In Environment and plants: Glimpses of research in South Asia (Eds. P.K Jha, R.P. Chaudhary, S.B. Karmacharya and V. Prasad). Ecological Society, Kathmandu, Nepal. pp. 264-274.

Joshi, A.R. 1979. Contribution to our knowledge on Myxophyceae of Nepal. J. Nat. Hist. Mus. (Nepal) 3(2): 35-41.

Komarek, J. and J. Lukavsky 1988. Arthronema, a new cyanophyte genus from Afro-Asian deserts. Arch. Hydrobiol. Suppl. Algol. Stud. 80: $249-267$.

Komarek, J. and M. Watanabe 1990. Morphology and taxonomy of the genus Coleodesmium (Cyanophyceae / Cyanobacteria). In Cryptogams of the Himalayas, Vol. 2, Central and Eastern Nepal (Eds. M. Watanabe and S.B. Malla). National Science Museum, Tsukuba, Japan. pp. 1-22.

Komarek, J. and M. Watanabe 1998. Contribution to the attached Cyanoprokaryotes from submerged biotopes in Sagarmatha National Park (Eastern Nepal). Bull. Natn. Sci. Mus., Ser. B, Tokyo. 24(4): 117-135.

Kusel-fetzmann, E. 1969. Einige Algen aus Nepal. Khumbu Himal (Berlin) 1(6): 37-56.

Nakanishi, M. 1986. Limnological study in Phewa, Begnas and Rupa lakes. In Studies on distribution, adaptation and evolution of microorganisms in Nepal Himalayas (Ed. Y. Ishida). (Second report), Ministry of Education, Science and Culture, Kyoto, Japan. pp. 3-13. 
S.K. Rai, R.K. Rai and S. Jha / Our Nature (2010) 8: 336-354

Prasad, R.C. and B.N. Prasad 2001. Screening of blue green algae (Cyanobacteria) and their distributional pattern in rice field of Narayani and Bagmati zones of Nepal. J. Liv. World $8(1): 1-12$.

Prasad, V. 1996. Blue green algae of Birganj (Nepal). Short term project. Research Division, Tribhuvan University, Nepal.

Rai, S.K. and P.K. Misra 2010. Freshwater cyanophyceae from east Nepal. Bangladesh J. of Plant Taxonomy 17(2): 121-139.

Sahay, A.P., P.K. Das and B.N. Verma 1993. Studies on the algal flora of Nepal-II: Cyanophyceae and Euglenophyceae. Geophytology 23(1): 181-183.

Shrestha, B. and J.D. Manandhar 1983. Contribution to the algal flora of Kathmandu valley. J. Inst. Sci. Techn. (Nepal) 6: 1-6.

Singh, R.N. 1961. Role of blue-green algae in nitrogen economy of Indian agriculture. I.C.A.R, New Delhi, India.

Subba Raju, N. and M.R. Suxena 1979. Algae and testacea of the Cho Oyu (Himalayas) Expedition-II; cyanophyta, chlorophyta, euglenophyta, chrysophyta and testacea. Hydrobiologia 67(2): 141-160.

Upadhyaya, B.N. 1979. Two new records of
Oscillatoria for Nepal. J. Nat. Hist. Mus.

(Nepal) 3(3): 74-75.

Watanabe, M. 1995. Algae from Lake Rara and its vicinities, Nepal Himalayas. In Cryptogams of the Himalayas, Vol. 3, Nepal and Pakistan (Eds. M. Watanabe and H. Hagiwara). National Science Museum, Tsukuba, Japan. pp. 1-17.

Watanabe, M. and J. Komarek 1988. Blue-green algae from Kathmandu. In Cryptogames of the Himalaya, Vol. 1, The Kathmandu valley (Eds. M. Watanabe and S.B. Malla). National Science Museum, Tsukuba, Japan. pp. 1-20.

Watanabe, M. and J. Komarek 1994. Several Cyanoprokaryotes from Sagarmatha National Park, Nepal Himalayas. Bull. Natn. Sci. Mus., Ser. B, Tokyo. 20(1): 1-31.

Yoshimura, Y., S. Kohshima and S. Ohtani 1997. A community of snow algae on a Himalayan glacier: change of algal biomass and community structure with altitude. Arctic and Alpine Research 29(1): 126-137.

Yoshimura, Y., S. Kohshima, N. Takeuchi, K. Seko and K. Fujita 2006. Snow algae in a Himalayan ice core: new environmental markers of ice-core analyses and their correlation with summer mass balance. Annals of Glaciology 43: 148-153. 